\title{
Thermal imaging of synchrotron beams on silicon crystals
}

\author{
by R. K. SMITHER $\left({ }^{*}\right)(*)$
}

(*) Advanced Photon Source, Argonne National Laboratory, Argonne, IL 60439 USA

\begin{abstract}
The Advanced Photon Source, a next generation synchrotron source, currently under construction at Argonne National Laboratory, will deliver large thermal loads of 1 to $10 \mathrm{~kW}$ to the first optical elements (usually a silicon crystal) in the synchrotron, X-ray beam lines. The first optical elements will distort and attenuate the $X$-ray beam if they are not extremely well cooled. An infrared camera is used to monitor the temperature distribution of the these first optical elements. This measurement is complicated because the silicon crystal is transparent to the infrared radiation and requires a special approach to the analysis of the data to get a meaningful temperature for the crystal.
\end{abstract}

\section{Introduction}

The high-brilliance insertion-device-based $X$-ray beams of the next generation of synchrotron sources will deliver large thermal loads to the first optical elements in the synchrotron beam lines. The first optical element will often absorb virtually all of the heat load of these intense X-ray beams, which will range in power from 1 to $10 \mathrm{~kW}$. The cooling of the first optical element under these extreme conditions becomes a serious problem, particularly when the first optical element is a diffraction crystal whose surface must be controlled to a few arc seconds. In order to help solve this high-heat-load problem, the experimental facilities group at Argonne National Laboratory (ANL) has undertaken a study of the use of different cooling geometries and different coolants for the first optical elements. The cooling fluid that showed the most promise for meeting these requirements was liquid gallium. Its high thermal conductivity, high specific heat and very low vapor pressure made it an excellent cooling fluid for this application and is especially advantageous for use in a highvacuum environment. Cooling experiments were performed at the synchrotron facilities at both Cornell University (CHESS) and at Brookhaven (NSLS) using a twocrystal monochromator. The temperature distributions on the surface of the first crystal were monitored with an infrared camera. These temperature distributions were quite useful in comparing the cooling efficiency of different cooling geometries and different cooling fluids as a function of flow rate and heat load. The infrared data were compared with calculated values based on the known distribution of heat in the synchrotron beam. Both the shape and the relative values of the temperature distribution in the infrared camera image agreed with the calculations, but the absolute values did not agree. There were some problems in interpreting the infrared data because the silicon crystals are highly transparent to the infrared radiation. This became a serious problem as the thickness between the cooling channels and the top surface of the crystal was reduced to dimensions of less than $1 \mathrm{~mm}$.

$\left({ }^{\star \star}\right)$ This work is supported by the U.S. Dept. of Energy, BES-Materials Science under contract No. W-31109-ENG-38. 


\section{Experimental configuration}

Figure 1 shows the experimental set up for the experiments performed at CHESS, the synchrotron facility at Cornell University. The $X$-ray beam emitted by the insertion device on the left, impinges on the first crystal of a two-crystal monochromator. A small fraction of the beam is diffracted upward and then is diffracted for a second time by the second crystal, which makes the direction of the diffracted $X$-ray beam level so that it will enter the experimental area in the same place as the monochromator is adjusted for diffracting different $X$-ray energies. Figure 2 show the typical position of the infrared camera during the experiment. The monochromator is housed in an aluminium box filled with helium. The infrared radiation passes through a very thin layer of plastic before entering the camera. The surface of the crystal is typically $10 \mathrm{~cm}$ in diameter. Figure 3 shows the power distribution, perpendicular to the beam, for a CHESS wiggler source. Gamma*Theta and Gamma*Psi are angular position coordinates given in mRad; in the horizontal and vertical directions, respectively. When the Advanced Photon Source (APS) is operating in 1995 , peak power densities of $100 \mathrm{~W} / \mathrm{cm}^{2}$ are expected for some of the wiggler beam lines. The X-ray beam will strike the surface of the diffraction crystal at an angle and will be spread out by a factor of 5 to 20 depending on the Bragg diffraction angle, or for an asymmetric cut crystal or inclined crystal, depending on the incident angle relative to the surface of the crystal.

\section{Experimental data}

Figure 4 shows the cross-section of the two types of silicon crystals used in the wiggler experiments at CHESS [1]. The upper cross-section shows the core-drilled crystal where the diameter of the circular channels is $2.4 \mathrm{~mm}$, and the thickness of the top layer above the channels is $1.2 \mathrm{~mm}$. The over-all size of the diffraction face of the crystal is $7.5 \mathrm{~cm}$ by $7.5 \mathrm{~cm}$. The gallium flows through the circular channels the full length of the crystal in the direction of the beam and covers a $5 \mathrm{~cm}$ width of the crystal in the direction perpendicular to the beam. In the second type of diffraction crystal, the gallium flows up through the base of the crystal on one side, across the crystal through small rectangular channels, $0.76 \mathrm{~mm}$ wide and $3 \mathrm{~mm}$ deep, and then down through the base of the crystal (see figure 5 ). The thickness of the silicon crystal above the rectangular channels was $0.61 \mathrm{~mm}$. Figure 6 shows the temperature isotherms for the wiggler beam incident on the core-drilled crystal. Figure 7 shows the cross-section of the temperature profile taken along the line $A-A^{\prime}$ which is parallel to the direction of the $\mathrm{X}$-ray beam. Figure 8 shows the cross-section of the temperature profile along line BB', perpendicular to the direction of the beam. The data points (solid circles) in figures 7 and 8 correspond to the isotherms shown in figure 6 . Note the wings or skirts on the peak in figure 7 . The shape of the incident power distribution is close to Gaussian in the direction of the beam. The wings are the result of heat flowing along the surface, away from the hot central region. This data was taken at CHESS with the $X$-ray beam from the 24 pole wiggler with an $85 \mathrm{~mA}$ beam current in the storage ring. The peak intensity in the $X$-ray beam was $12.3 \mathrm{~W} / \mathrm{mm}^{2}$ on a surface perpendicular to the beam. In this experiment, the incident angle of the $X$-ray beam on the face of the crystal was 10.36 degrees. This angle causes the footprint of the beam to be spread out by a factor of 5.56 . This spreading out reduces the peak power on the surface of 
the crystal to $2.21 \mathrm{~W} / \mathrm{mm}^{2}$.

Similar data was taken with the slotted crystal and the maximum temperature difference between the peak temperature and the surrounding unheated crystal as a function of flow rate of the liquid gallium through the channels is plotted for both crystals in figure 9 . Each curve is labeled with a storage ring beam current that can be used to obtain the peak power density as described above. A Inframetrics 525 infrared camera was used in these experiments. The temperature reading were calibrated by comparing their signals to those of a thick piece of silicon for which the temperature was known and could be controlled to match the crystal infrared signal strength. The slotted crystal did a much better job of cooling the crystal and exhibited much lower temperatures for similar flow rates. The temperatures of both crystals were decreasing with increased flow rates so increased flow should decrease the temperatures even further.

\section{Interpretation of data}

The use of infrared data in these studies is very useful in that it separates out the temperature effect from other kinds of effects that could affect the diffraction of the X-ray beam such as vibrations and / or distortions of the crystal. If one were only monitoring the $X$-ray flux out of the two-crystal monochromator, the increased cooling due to increased flow of gallium could be masked by an increase in the vibrations present in the crystal due to increased flow or distortions of the crystal arising from the increased pressure in the crystal needed to increase the flow of gallium. The main uncertainty in how one should interpret the infrared data comes from the fact that silicon is highly transparent to infrared radiation in just the energy range where the infrared is sensitive. Thus, the actual infrared reading is some kind of average of the radiation being emitted from the whole top layer of silicon above the liquid gallium channels. Some of the various kinds of sequences of events over which one would have to integrate to obtain this average are shown in figure 10a for a simple slab of silicon and in figure $10 b$ for the slotted crystal. If the absorption of the infrared radiation is quite low, as it is in silicon, then the photons can be reflected many times as they bounce back and forth between the front and back surface of the crystal. The general form of the equation for the radiation that escapes from the crystal is given by:

$$
I(\text { out })=I_{o} T_{o}(1-R)\left[1+T^{2} R^{2}+T^{4} R^{4}+T^{6} R^{6}+\ldots\right]
$$

where $I_{0}$ is the initial flux, $T_{0}$ is transmission fraction or one minus the absorption of the radiation as it travels from the origin of the radiation to the first surface that it encounters, $T$ is the transmission fraction as the radiation travels from one surface to the next, and $R$ is the reflection coefficient at the surface. If the two absorption terms, $T_{O}$ and $T$, are so low that it can be ignored, then equation (1) becomes

$$
I(\text { out })=I_{0}(1-R)\left[1+R^{2}+R^{4}+R^{6}+\ldots . .\right]
$$

The reflection coefficient at these wavelengths is approximately 0.3 . Substituting this value in equation (2) we have 
http://dx.doi.org/10.21611/qirt.1992.046

$$
I(\text { out })=I_{0}(0.7)[1+0.09+0.0081+0.00073+\ldots]=0.769 I_{0}
$$

If 10 percent of the radiation were to be absorbed on each pass through the crystal, $T=0.9$ and $T_{0}=0.95$, then the value of $I$ (out) would be slightly lower,

$$
I(\text { out })=I_{0}(0.95)(0.7)[1+0.073+0.0053+0.00039]=0.717 I_{0}
$$

This value comes quite close to the calibration value obtained with $1.5 \mathrm{~cm}$ thick silicon crystals. The thin top layers of our crystals should be closer to the zero absorption case given in equations (2) and (3). To the first approximation, the effective emissivity of the crystal is equal to the transmission coefficient at the surface or (1-R). The presence of channels filled with gallium will complicate this analysis to some degree. Figure $10 \mathrm{~b}$ illustrates this complication. In practice, the gallium acts like a metallic, highly reflective surface and looks very similar to the sources near it. The ribs between the slots act like radiation traps, slightly reducing the radiation in this region. In the slotted crystal, this variation can be seen when the crystal is viewed with the IR camera with no beam on the crystal as shown in figure 11. The effect of the channels can be seen in the core-drilled crystal as well, but their effect on the apparent temperature is considerably less, a variation of only $0.5^{\circ} \mathrm{C}$ in contrast to the $2.5^{\circ} \mathrm{C}$ variation seen with the slotted crystal. This last data was taken with a Inframetrics 725 camera, see color figure $A^{*}$ which has better resolution than the 525 camera.

\section{Conclusions}

Approximately the right temperatures are measured with the IR camera if one uses an emissivity coefficient of 0.7 and accepts an uncertainty of about 10 percent on the difference between two temperatures. The temperature that one observes is an average temperature for the full top layer of silicon crystal above the gallium-filled channels.

\section{REFERENCES}

[1] SMITHER (R.K.), LEE (W.), MACRANDER (A.), MILLS (D.) and ROGERS (S.). - Recent experiments with liquid gallium cooling of crystal diffraction optics.

Rev. Sci. Instrum., 6 3, 1992, p. 1746-1754. 

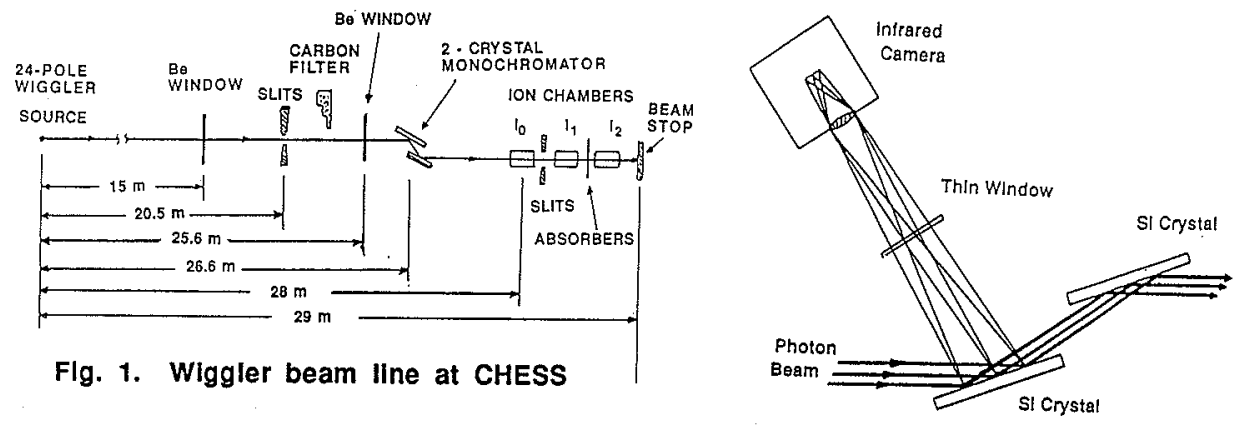

FIg. 2. Infrared camera set up

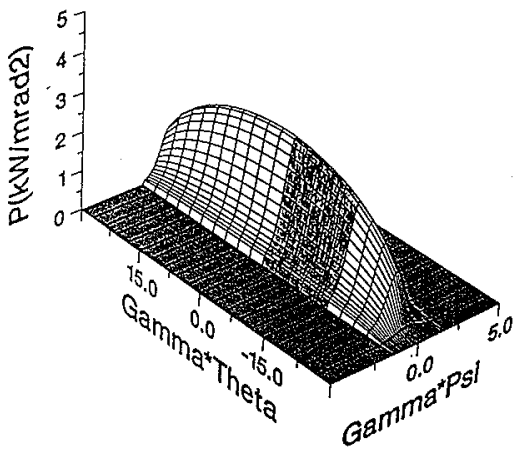

Fig. 3. Power distribution from wiggler

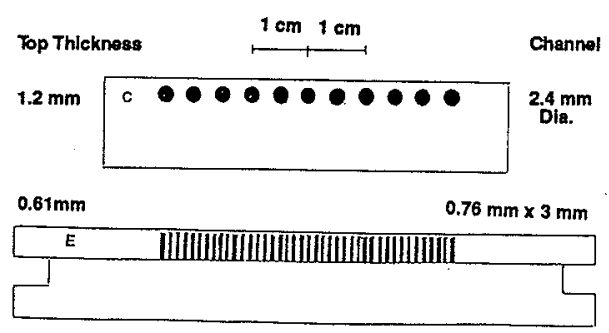

Fig. 4. Cross-section of two crystals used

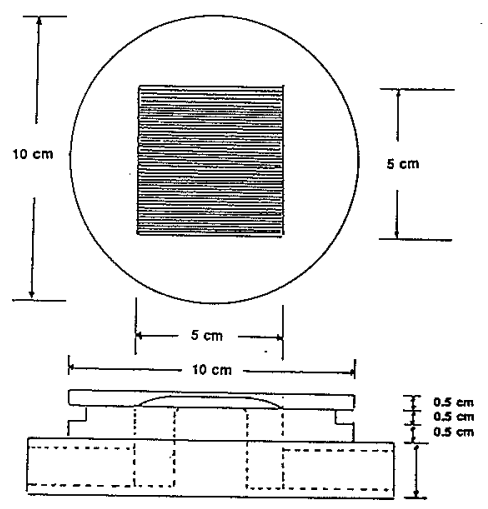

Fig. 5. Vlews of slotted crystal

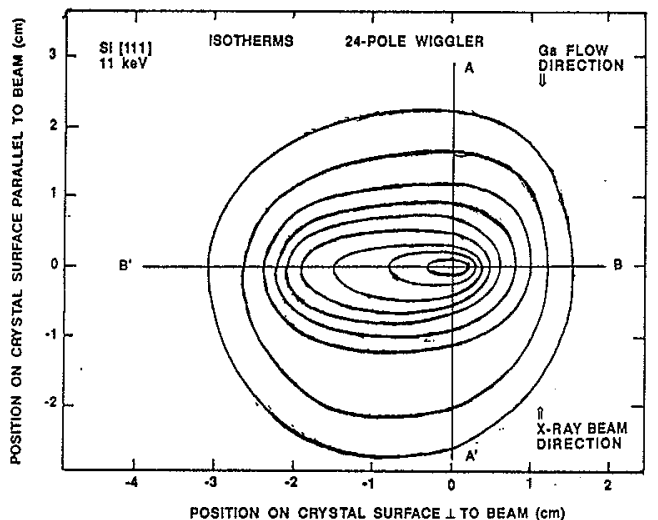

Fig. 6. Isotherms on core-drllled crystal 


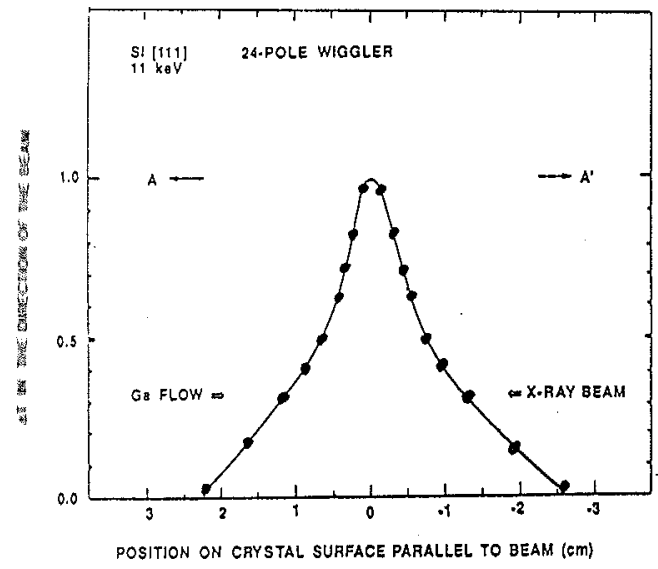

Fig. 7. Cross-section of the thermal proflle laken alone the line A-A'

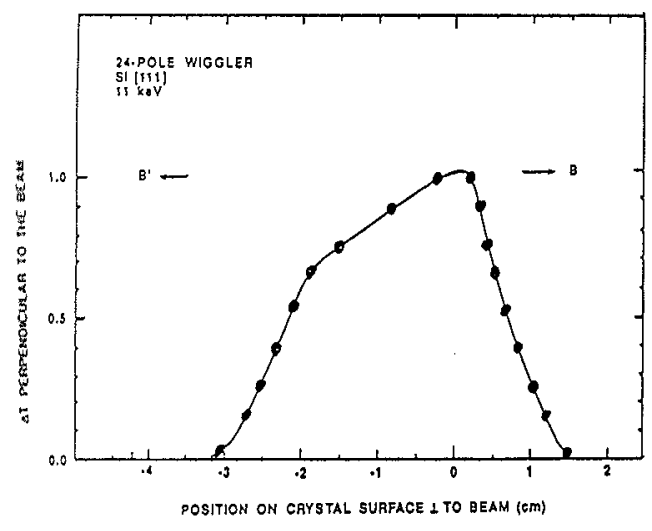

Flg. 8. Cross-section of the thermal proflle taken alone the line B-B'

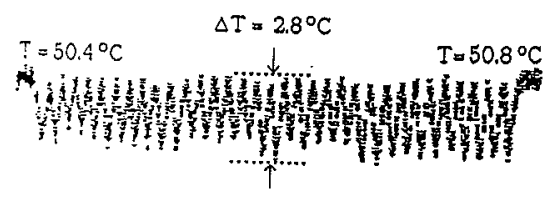

FIg.11. Temperature proflle perpendicular to coollng slots

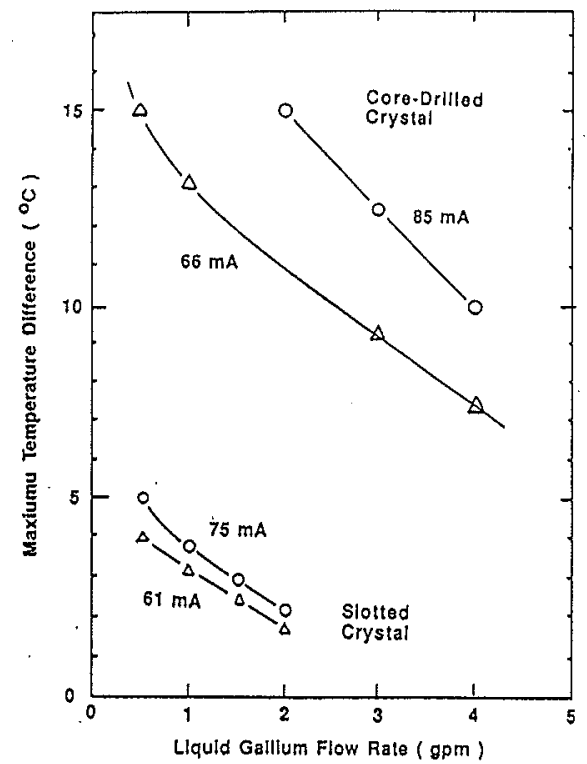

Fig. 9. Max. temperature difference for core-drilled and slotted crystals

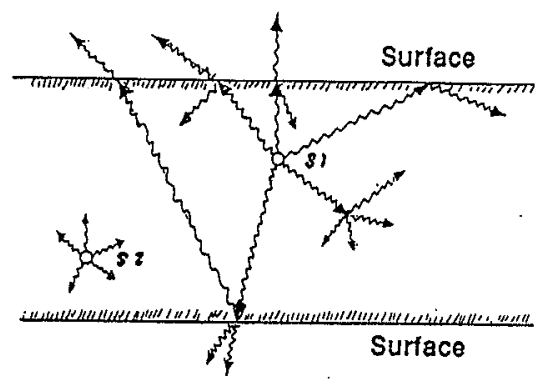

FIg.10a. Radlation from a uniform plate

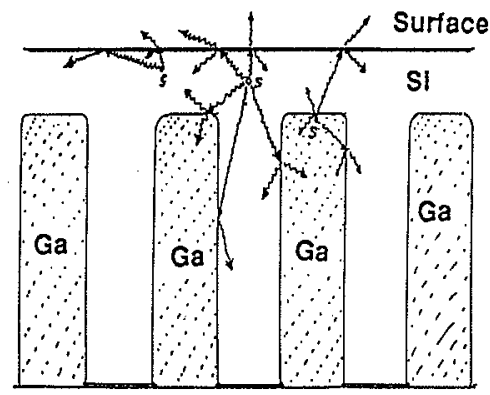

SI

Fig.10b. Radlation from a slotted crystal 